\title{
Philosophiques
}

\section{La naissance de la théorie des types}

\section{François Lepage}

Volume 11, numéro 2, octobre 1984

Égalité, justice et différence

URI : https://id.erudit.org/iderudit/203258ar

DOI : https://doi.org/10.7202/203258ar

Aller au sommaire du numéro

Éditeur(s)

Société de philosophie du Québec

ISSN

0316-2923 (imprimé)

1492-1391 (numérique)

Découvrir la revue

Citer cet article

Lepage, F. (1984). La naissance de la théorie des types. Philosophiques, 11(2), 277-297. https://doi.org/10.7202/203258ar

\section{Résumé de l'article}

La théorie des types que Bertrand Russell proposa en 1908 ne se voulait pas une solution ad hoc au problème des contradictions, elle prétendait plutôt être la solution naturelle, celle que tout le monde reconnaîtra comme la solution attendue. En fait, il s'agit d'une théorie philosophique qui concrétise un projet grandiose: réduire les mathématiques à la logique. Le présent texte se propose d'examiner les thèses russelliennes et la dynamique de leur évolution de 1903 à 1907, c'est-à-dire des Principles à la naissance de la théorie des types.
Ce document est protégé par la loi sur le droit d'auteur. L'utilisation des services d’Érudit (y compris la reproduction) est assujettie à sa politique d'utilisation que vous pouvez consulter en ligne.

https://apropos.erudit.org/fr/usagers/politique-dutilisation/ 


\title{
LA NAISSANCE DE LA THÉORIE DES TYPES
}

\author{
par François Lepage
}

RÉSUMÉ. La théorie des types que Bertrand Russell proposa en 1908 ne se voulait pas une solution ad hoc au problème des contradictions, elle prétendait plutôt être la solution naturelle, celle que tout le monde reconnaîtra comme la solution attendue. En fait, il s'agit d'une théorie philosophique qui concrétise un projet grandiose : réduire les mathématiques à la logique. Le présent texte se propose d'examiner les thèses russelliennes et la dynamique de leur évolution de 1903 à 1907, c'est-à-dire des Principles à la naissance de la théorie des types.

ABSTRACT. The theory of types that Bertrand Russell proposed in 1908 didn't present itself as an ad hoc solution to the problem of contradictions. Rather it pretended to be the natural solution, the one that everybody would recognize as the expected solution. In fact, it is a philosophical theory materializing an imposing project : to reduce mathematics to logic. This paper examines the russellian theses and their evolution from 1903 to 1907 i.e. from the Principles to the birth of the theory of types.

Lorsqu'en 1908, dans son fameux article "Mathematical Logic as Based on the Theory of Types", Russell expose la théorie des types, son intention n'est pas seulement d'avancer une méthode permettant de rebâtir les mathématiques tout en évitant les contradictions. Ce que Russell propose se veut la solution. En effet, pour son auteur, la nouvelle théorie a non seulement le mérite d'éviter les contradictions, mais elle a aussi " une certaine consonnance avec le sens commun qui la rend en soi crédible ${ }^{1}$.

Le présent texte a pour objectif d'analyser cette crédibilité que lui prête son auteur en examinant les racines philosophiques de la théorie des types. Car, curieusement, cette crédibilité aurait

1. In Logic and Knowledge, London, 1956, p. 59. 
plutôt pour effet, à première vue, de rendre cette théorie suspecte. Dirions-nous, par exemple, que la théorie des groupes est crédible ? La notion de crédibilité s'appliquerait plutôt à une théorie à contenu empirique. Si la théorie des types est crédible, c'est que, pour son auteur, elle dit quelque chose de crédible à propos de quelque chose d'autre. En l'occurrence, la théorie des types est une théorie sur l'être.

Nous n'avons pas l'intention ici d'exposer la théorie des types, de discuter ses propriétés formelles ou de sa capacité à exprimer les mathématiques. Notre but, plus modeste, est d'explorer ou du moins de semer des jalons pour l'exploration de la pensée qui a permis l'élaboration d'une telle théorie.

Pour ce faire, nous avons volontairement restreint notre attention à certains textes de Russell, écrits entre 1900 et 1906 (mis à part deux textes à caractère biographique où Russell parle de cette période). Cette restriction exige cependant une certaine justification.

Tout d'abord, la notion de type apparaît pour la première fois dans l'appendice B des Principles of Mathematics, ouvrage paru en 1903 mais qui a été presque entièrement rédigé en 1900, soit avant la découverte de la contradiction ${ }^{2}$. Cet ouvrage et les thèses philosophiques qu'il contient recevront donc une attention particulière. D'autre part, nous nous sommes limité aux textes d'avant 1908, c'est-à-dire aux textes qui précèdent la première formulation complète des principes de la théorie des types. Ces deux dates nous semblent en effet fixer les bornes de la période de genèse de la théorie, période où le choix du cadre théorique global n'est pas définitivement arrêté et donc où toutes les directions peuvent s'avérer de bonnes directions.

On remarquera aussi qu'il ne sera à peu près pas question de Frege dans ce qui suit. Cela peut sembler à première vue insolite car il est souvent fait référence à Frege dans les textes de Russell de la période qui nous intéresse. Nous croyons cependant que la discussion et l'interprétation des thèses frégéennes ne sont pas essentielles à la compréhension de la philosophie de

2. Voir par exemple Autobiography, Londres, 1967. Traduction française par M. Berveiller (ciaprès $A$.). 
Russell, et qu'au contraire, la recherche d'un parallélisme entre ces deux pensées peut être génératrice de confusion. Malheureusement, la justification de cette prise de position exigerait un travail d'une ampleur au moins égale à celle de l'analyse qui va suivre. Nous nous contenterons donc de renvoyer le lecteur à la littérature pertinente ${ }^{3}$.

\section{GRAMMAIRE ET ONTOLOGIE}

S'il faut fixer un point de départ à la théorie des types, on peut dire, en suivant les indications de Russell lui-même, que son histoire commence en 1900, au congrès international de philosophie de Paris, où Russell rencontre Peano ${ }^{4}$. La précision avec laquelle celui-ci traite de problèmes fondamentaux impressionne Russell. Un grand projet va prendre forme, celui de réduire les mathématiques à la logique. Cette entreprise comportera deux étapes. Premièrement, Russell se propose d'exposer et de justifier la méthode qu'il va utiliser et deuxièmement, de réaliser effectivement cette réduction.

La première partie sera l'objet de son ouvrage The Principles of Mathematics, qui devait être le premier de deux tomes (le second ne verra jamais le jour $)^{5}$.

Cette entreprise de réduction des mathématiques à la logique peut schématiquement se résumer ainsi :

\footnotetext{
Dynamique $\longrightarrow$ analyse $\longrightarrow$ arithmétique $\longrightarrow$ logique $\longrightarrow$ grammaire philosophique ${ }^{6}$.
}

3. Voir Searle, J.R. "Russell's Objections to Frege's Theory of Sense and Reference", in Analysis, 18, (1958), p. 137-142. Également, Cassin, C.E., "Russell's Discussion of Meaning and Denotation : A Re-examination", in Essays on Bertrand Russell, Klemke, E.D. (ed.), Chicago, 1971, p. 256-272. Je me permettrai cependant de citer Geach, P.T., qui en conclusion à "Russell on Meaning and Denoting", in Analysts, 19, (1958-59), p. 69. 72, écrit : "Considérés comme critiques non pas de Frege mais des propres théories de Russell dans $P M$ [Principles of Mathematics], les arguments de On Denoting acquièrent une force et une pertinence passablement différentes. Je n'essaierai pas ici de déterminer si elles sont valides. Il est regrettable que Russell, comme Aristote, déforme si souvent la pensée d'autrui dans son propre moule ; les lecteurs de $O n$ Denoting feront mieux d'ignorer tout simplement son utilisation du nom de Frege ».

4. Voir My Pbilosophical Development, London, 1959. Traduction française par G. Auclair, Paris 1961, (ci-après M.P.D.), p. 81, et A., p. 185-187.

5. Voir la préface des Principles of Matbematics, London, 1903, seconde édition avec une nouvelle introduction, 1937 , p. XV (ci-après $P$.).

6. Voir Wood, A., Essais sur l'évolution de la philosophie de Russell, publié en appendice à M.P.D., p. $325-326$. 
Or, si l'idée de réduire la dynamique à l'analyse, ou encore l'analyse à l'arithmétique, était déjà assez commune, celle de réduire la logique à la grammaire philosophique l'était moins. Cette entreprise mérite d'être examinée de plus près.

Les Principles commencent par une définition des mathématiques pures :

La mathématique pure est la classe de toutes les propositions de la forme " $p$ implique $q$ », où $p$ et $q$ sont des propositions contenant une ou plusieurs variables, les mêmes dans les deux propositions, et ni $p$ ni $q$ ne contiennent de constantes sauf des constantes logiques ${ }^{7}$.

La notion centrale sur laquelle repose toute la construction est celle d'implication.

Il y a deux sortes d'implications :

a) L'implication matérielle. L'implication matérielle est une relation ${ }^{8}$ qui a lieu entre deux propositions en autant que la première est fausse ou que la seconde est vraie.

b) L'implication formelle. L'implication formelle est une relation entre assertions. Or, l'explication de cette relation va nécessiter une analyse profonde des propositions :

Là où une implication formelle a lieu, nous avons convenu de la considérer, partout où c'est possible, comme étant due à quelque relation entre les assertions concernées. Cette théorie soulève plusieurs problèmes logiques redoutables, et requiert, pour sa défense, une analyse minutieuse des constituants des propositions?

Alors Russell va s'engager dans une exploration et une élucidation du monde de l'être, de la nature de la grammaire et des propositions. Nous nous contenterons ici de résumer l'essentiel du système russellien ${ }^{10}$. Le monde de l'être est composé

7. P., p. 3. Voir aussi p. VII-VIII.

8. Voir $P$., p. 34. On remarquera qu'aucune des deux sortes d'implications n'est considérée comme connecteur, mais bien comme relation.

9. P., p. 41.

10. Notre présentation du système de Russell est volontairement succincte. Pour une étude plus complète de la question, on consultera notre article : " La notion de proposition dans les Principles de Russell : un exemple de conception réaliste du langage », in Dialogue, vol. XVIII, no 4, 1979. 
de termes. Un terme étant n'importe quoi pouvant être l'objet d'une proposition.

Quoi que ce soit pouvant être un objet de pensée, ou avoir une occurrence dans n'importe quelle proposition vraie ou fausse, ou pouvant être compté comme un, je l'appelle un terme. C'est donc le mot le plus général du vocabulaire philosophique ${ }^{11}$.

Les termes sont inaltérables, éternels, etc. Ils correspondent à ce qu'on désigne habituellement sous le nom de substance. Ainsi, un terme ne peut être modifié par des attributs ou des relations. Un terme modifié est un autre terme. On voit ici poindre le problème qui hantera la philosophie de Russell jusqu'aux Principia : le fameux problème de l'un et du multiple ${ }^{12}$.

En effet, le monde de l'être, de par sa composition, semble irréductiblement multiple. L'inaltérabilité des termes rend difficile la justification d'une structure du monde de l'être. Une certaine unité, forme, etc. doit trouver une raison d'être quelque part, car il serait absurde de vouloir nier que l'être est structuré. Les propositions sont justement l'expression de cette structure. C'est ici qu'intervient la théorie des relations externes, théorie qui sera l'une des grandes constantes de la pensée de Russell tout en demeurant une source féconde d'inspiration ${ }^{13}$.

Cette théorie affirme quaucune relation ne modifie les termes qu'elle relie.

Bref, aucune relation n'a jamais modifié aucun de ses termes.

Car si elle a lieu entre $A$ et $B$ c'est entre $A$ et $B$ qu'elle a lieu, et dire qu'elle modifie $A$ et $B$ c'est dire qu'elle a vraiment lieu entre des termes différents $C$ et $D^{14}$.

C'est donc cette théorie qui servira de cadre pour l'expression de la structure de la proposition. Russell distingue d'abord trois sortes de termes ${ }^{15}$.

Termes $\left\{\begin{array}{l}\text { Choses } \\ \text { prédicats } \\ \text { relations }\end{array}\right\}$ concepts

Une proposition se compose de termes parmi lesquels on

11. P., p. 43.

12. Voir les commentaires de Quine, V.W., "Russell's Ontological Development" in Klemke, op. cit., p. 3-14.

13. Voir Winslade W.J., "Russel's Theory of Relations", in Klembe, op. cit., p. 81-101.

14. P., p. 448.

15. Voir $P$., p. 48. 
trouve une relation et c'est cette relation qui est responsable de l'unité de la proposition. Comme ni la relation ni les termes ne sont modifiés, nous appellerons cette théorie la théorie combinatoire de la proposition.

Nous nous devons maintenant de dire quelques mots sur la nature de la grammaire.

Le monde de l'être et sa structure nous sont accessibles par le langage. Ainsi, aux trois sortes de termes correspondent trois catégories grammaticales : nom, verbe et adjectif.

On a plus ou moins un isomorphisme entre la structure propositionnelle (la structure du monde de l'être) et la structure grammaticale.

On peut résumer la théorie de Russell par le schéma suivant :

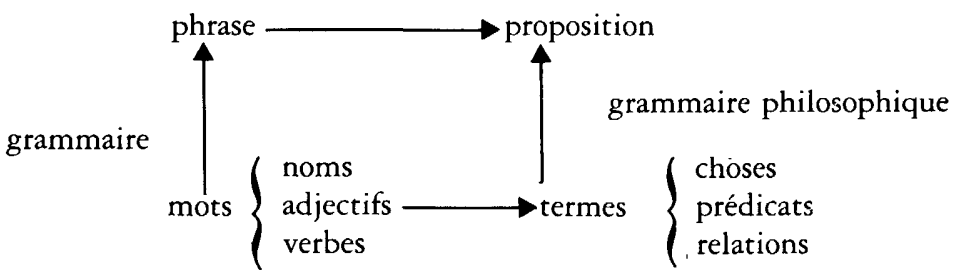

La théorie russellienne pose plusieurs problèmes difficiles. Parmi ceux-ci, celui de la dénotation occupe une place importante $^{16}$.

Certains mots, dans certains contextes, semblent renvoyer à des entités complexes, par exemple " tous les hommes ». Russell développe une théorie compliquée d'où il ressort que les classes peuvent parfois être considérées comme des objets, parfois comme des multiplicités. Dans « tous les hommes sont mortels », pour reprendre notre exemple, la théorie de Russell nous conduirait à analyser la proposition correspondante comme ayant les concepts hommes et mortels comme constituants. Or, ce n'est pas le concept d'homme qui est mortel mais les termes qu'il subsume.

16. Voir Cassin, C.E., "Russell's Discussion of Meaning and Denotation : A Re-examination", in Klemke, op. cit., p. 257-258. Voir aussi Jager, R., The Development of Bertrand Russell's Philosophy, Londres, 1972, p. 146. 
Ce problème est d'autant plus aigu qu'il concerne la notion de classe, notion d'une importance cruciale dans la perspective fondationnelle où se situe Russell.

Une classe a ainsi un double aspect : en un sens, elle est une unité, en un autre elle est une multiplicité. Les notions et les concepts iront donc par paires que nous mettons ici en opposition :

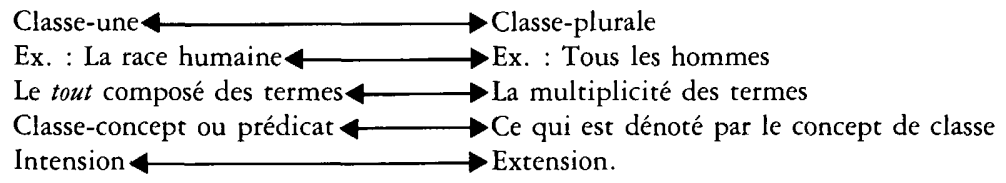

Russell développe alors une méfiance à l'égard des classes, méfiance dont, nous le verrons plus loin, on ne saurait négliger l'impact. Et la découverte de la contradiction ne va pas arranger les choses.

\section{LA CON'TRADICTION}

En examinant la preuve de Cantor « qu'étant donnée une classe quelconque (finie ou infinie), la classe de toutes les sousclasses de la classe donnée possède strictement plus d'éléments que celle-ci », Russell découvre une contradiction : la classe de toutes les classes qui ne s'appartiennent pas, s'appartient et ne s'appartient pas $^{17}$.

Russell s'aperçoit très tôt que la contradiction est universelle en ce sens qu'elle n'est pas liée à des engagements ontologiques spécifiques. On peut en effet exprimer la contradiction en termes de prédicat, en termes de classe-concept, en termes classe-une et classe-plurale. Russell envisage plusieurs façons d'éviter cette contradiction. Une chose est claire cependant :

La raison pour laquelle une contradiction apparaît est que nous avons considéré comme un axiome que toute fonction propositionnelle contenant une seule variable est équivalente à l'assertion

17. Du moins, c'est ce que raconte Russell à plusieurs endroits. Par exemple, dans son Autobiographie, dans son Introduction à la philosophie mathématique et dans son Histoire de mes idées philosopbiques. Il semble que la réalité soit beaucoup plus complexe et que "l'accouchement \#, de la contradiction ait été assez laborieux. Voir à ce sujet Coffa, J.A., "The humble origins of Russeli's paradox" in Russell : The Journal of Bertrand Russell Archives, p. 33-34, (1979). 
de l'appartenance à une classe définie pat la fonction propositionnelle ${ }^{18}$.

Russell voit donc très bien que ce qui est en jeu est ce qu'on appelle classiquement le principe de compréhension. C'est ce principe qu'il faudra restreindre. La voie que nous retiendrons parmi celles que propose Russell est celle de la théorie des types logiques publiée en appendice des Principles.

\section{LA THÉORIE DES TYPES DES PRINCIPLES}

La théorie des types de 1903 propose une façon simple de restreindre le principe d'extensionnalité. Ainsi, à chaque fonction propositionnelle est associé un domaine de signification qui est composé de tous les objets qui, lorsque pris comme arguments par la fonction propositionnelle, donnent des propositions vraies ou fausses. Les domaines de signification les plus petits sont appelés types minimaux ${ }^{19}$. Les types forment une hiérarchie : tout d'abord, il y a les individus qui composent le type le plus bas, un individu étant un objet qui n'est pas un domaine, suivent les classes d'individus qui forment le type I, puis les classes de classes, etc.

Comment cette théorie peut-elle résoudre la contradiction? La réponse est simple :

. . . non- $u$ doit être le domaine de fausseté de « $x$ est un $u$ », et " $x$ est un $x$ " doit être en général dépourvu de signification (meaningless) ; conséquemment, " $x$ est un $u$ » doit exiger que $x$ et $u$ soient de types différents ${ }^{20}$.

Cette remarque de Russell contient une expression qui résume toute l'ambiguiité et toute la problématique de la théorie des types de 1903 : l'expression «en général ». Qu'est-ce que cela peut bién signifier?

Russell lui-même remarque que sa théorie ne permet d'éviter la contradiction que si l'on se borne à considérer des types

18. P., p. $102-103$.

19. La distinction que fait Russell entre un type et un domaine de signification est assez subtile. Un domaine de signification est soit un type, soit la réunion de plusieurs types $(P .$, p. 523). Mais comme un type quelconque est la réunion de types minima (p. 524), on doit conclure qu'un domaine de signification est un type.

20. P., p. 524. 
minima. Or, ce n'est pas un hasard si Russell a besoin de types mixtes (qui sont la réunion de types minimaux) et même de classes dont les éléments doivent être du même type qu'elles. En effet, pour Russell la classe-une est un individu si ses éléments sont des individus et, en général, une classe-une d'objets d'un type quelconque est de ce type.

On peut justifier cette prise de position qui, on le remarquera, détruit le point essentiel de la théorie des types en regard de la contradiction, de la façon suivante. Nous avons vu que l'entreprise de Russell consiste en l'élaboration d'une combinatoire logique qui rendrait compte de la structure du réel. Une de ces combinatoires élémentaires est le regroupement d'objets en classes pour former d'autres objets. Par exemple, une personne est une classe d'existants psychiques et une pomme est une classe de points matériels ${ }^{21}$. Alors, si dans la théorie il fallait distinguer entre l'humanité, qui est pour Russell un objet bien défini, et un homme au point de vue typologique, nous nous engagerions dans une régression que nous ne serions pas sûr de pouvoir arrêter. Autrement dit, comme les objets pour lesquels nous avons des noms ne sont (presque) jamais simples, ils doivent être du même type que leurs composants, sinon il est vraisemblable que nous ne pourrions pas déterminer à quel type ils appartiennent. Et ainsi, c'est toute la théorie du langage des Principles qui s'écroule. Bref, la théorie des types de 1903, même si elle semble prometteuse en ce qui concerne l'élimination de la contradiction, ne peut pas s'intégrer à la philosophie du langage des Principles.

D'autres considérations viennent de plus augmenter le doute de Russell face à l'utilité d'une théorie des types aussi stricte. En effet, la théorie du langage des Principles se veut universelle et dans une théorie des types stricte, il n'est pas possible de parler des types (de quel type sont les types ?). Or « . . . tous les domaines forment certainement un type, vu que chaque domaine a un nombre (has a number), et de même tous les objets, vu que chaque objet est identique à lui-même ${ }^{22}$. Le problème se pose donc de façon très claire : la théorie des types de 1903 permet d'éviter la contradiction mais le prix à payer est très

21. P., p. 523 .

22. P., p. 525 . 
grand, trop grand. Il n'est plus possible de formuler des propositions universelles, c'est-à-dire des propositions contenant des variables liées qui soient vraies en vertu de leur forme seulement et donc indifférentes au domaine de valeurs associé à ces variables. Or, cette condition est fondamentale pour l'entreprise de fondement des mathématiques telle que Russell la conçoit à cette époque. Nous avons vu, en effet, que pour Russell les mathématiques sont la classe des implications entre propositions ne contenant que des constantes logiques. Plus explicitement,

Nous assertons toujours en mathématiques que si une certaine assertion $p$ est vraie d'une certaine entité $x$, ou d'un certain ensemble d'entités $x, y, z, \ldots$, alors une certaine autre assertion $q$ est vraie de ces entités ; mais nous n'affirmons ni $p$ ni $q$ de nos entités séparément. Nous assertons une relation entre les assertions $p$ et $q$, que j'appellerai implication formelle ${ }^{23}$.

Mais justement, il est de la nature même de l'implication formelle qu'il n'y ait pas de restriction sur le domaine des valeurs de la variable. Là-dessus, Russell est on ne peut plus strict.

Nous devons donc permettre à notre $x(\ldots)$ de prendre toutes les valeurs sans exception; et là où une restriction sur la variabilité est exigée, l'implication ne doit pas être considérée comme formelle jusqu'à ce que ladite restriction ait été levée en étant préfixée en tant qu'hypothèse $\mathrm{e}^{24}$.

La théorie des types de 1903 est donc inacceptable : le projet d'asseoir les mathématiques sur la logique, du moins à la façon dont Russell prétend le faire, n'y trouve pas les instruments suffisants. Cette approche théorique sera délaissée et ne ressortira pour s'épanouir que trois ans plus tard. Les questions que l'on est en droit de se poser sont les suivantes : tout d'abord, quelles modifications subira le système des Principles pour permettre d'exprimer une théorie des types ? Cette question en appelle une seconde plus intéressante : ces modifications se développeront-elles au fur et à mesure que la théorie des types s'imposera comme la solution aux paradoxes ou, au contraire, la théorie des types trouvera-t-elle un terrain d'épanouissement

23. P., p. 5.

24. P., p. 38 - Appliquer ce principe aux types reviendrait à remplacer une proposition mathématique $\mathrm{px} \rightarrow \mathrm{qx}$ par $\mathrm{Tx} \rightarrow(\mathrm{px} \rightarrow \mathrm{qx})$ où $\mathrm{Tx}$ signifierait « $\mathrm{x}$ est du type $\mathrm{T}$ ». Mais cela suppose que l'on puisse parler de type à l'intérieur de la théorie, ce qui soulève, comme nous l'avons vu, de graves difficultés. 
naturel dans le cadre d'une philosophie modifiée ? Il s'avère que la seconde partie de l'alternative fournit un cadre d'analyse plus intéressant.

Après la rédaction des Principles, Russell laisse ainsi de côté l'approche typologique. En 1959 il écrira :

Quand les Principes des Mathématiques furent terminés, je me mis résolument à chercher une solution à ces paradoxes. (. . .) de quelque façon que je m'y prisse, je ne faisais pas de progrès. En 1903 et 1904, j'ai consacré presque tout mon temps à cette question, mais sans le moindre succès. J'obtins un premier résultat avec la théorie des descriptions $(. . .)^{25}$.

Russell se trouve donc dans une impasse et le « sans le moindre succès » nous renseigne sur l'espoir qu'il investit dans sa théorie des types. D'ailleurs, ces allusions à l'impasse intellectuelle des années 1903-4 ainsi qu'au rôle qu'a joué la théorie des descriptions dans le dénouement de la crise sont fréquentes. ${ }^{26}$

\section{LA THÉORIE DES DESCRIPTIONS}

La théorie des descriptions sera donc, selon son auteur, le premier pas vers l'élimination des paradoxes. Examinons la chose d'un peu plus près ${ }^{27}$.

"On Denoting" (où Russell présente pour la première fois sa théorie des descriptions) débute par la distinction entre deux

25. M.P.D., p. 98-99.

26. Dans son Autobiograpbie, Russell écrira en 1967: "Chaque matin je m'asseyais devant une feuille blanche. Toute la journée, avec un bref entracte pour le déjeuner, je regardais cette feuille fixement. Souvent quand venait le soir elle était encore blanche. (. . .) En 1905 les choses commencèrent à s'améliorer. (. . . .) presque aussitôt je découvris ma théorie des descriptions, laquelle représentait la première étape vers la solution des difficultés qui m'avaient arrêté si longtemps. (. . .) En 1906, enfin, je découvris la théorie des types ". (On remarquera que Russell semble donc fixer la naissance de la théorie des types en 1906). Russell exagère quelque peu sans doute les effets inhibiteurs des problèmes dus à la contradiction : entre les Principles et "On Denoting", il a écrit dix articles ou comptes rendus concernant des problèmes de logique.

27. Mon interprétation de la théorie des descriptions met l'accent sur son contenu ontologique. Cette approche n'est pas entièrement originale. Voir par exemple Dufumier, H., "Les théories logico-métaphysiques de MM. B. Russell et G.E. Moore ", Revue de métaphysique et de morale, 1909. Nous l'avons développée ailleurs: voir notre article "Réalisme et théorie russellienne des descriptions ", in Canadian Journal of Philosophy, XIII, 2, (1983), 209-226. Notre interprétation se démarque de celle qui y voit une doctrine de la forme logique du langage, comme par exemple Kaplan, D. dans "What is Russell's Theory of Description" in Bertrand Russell, Pears, D.F. (ed.), New York, 1972, p. 227-244, et de celle qui y voit une théorie de la référence comme, par exemple, Linsky, L., Referring, Londres, 1967, traduction française par Stern-Gillet, S., Devaux, P. et Gochet, P., Paris, 1974. 
types de connaissances. La première est la connaissance "by acquaintance", que nous traduisons par " connaissance par fréquentation ". Bien que Russell ne donne pas d'exemples explicites de connaissance par fréquentation, on peut caractériser celleci par son caractère direct, immédiat.

Russell nous donne par contre des exemples explicites du deuxième type de connaissance, qu'il appelle « connaissance par description ". Le centre de masse du système solaire à un instant défini, l'esprit des autres personnes, l'actuel roi d'Angleterre, etc. Bref, chaque fois qu'une phrase utilise une expression dénotante (le, un, quelque, tous, etc.), ce sur quoi porte la proposition est l'objet d'une connaissance du deuxième genre.

Il semble bien qu'à cette époque - cela ne sera plus vrai plus tard - la différence entre les deux types de connaissance ne repose en aucune façon sur des différences entre les objets de connaissance. Le même objet peut ainsi, théoriquement, faire l'objet des deux types de connaissance. Bien que Russell ne prenne pas à cette époque position explicitement, on peut conclure que la différence grammaticale reflétant les deux types de connaissance est la suivante : la connaissance par fréquentation est indiquée par l'utilisation des noms propres, alors que la connaissance par description est indiquée par l'utilisation des expressions dénotantes. Ceci explique que la phrase "Scott est Scott " ne renvoie pas à la même proposition que "Scott est l'auteur de "Waverley" ». bien que Scott soit l'auteur de Waverley.

En quoi la théorie des Principles se trouve-t-elle modifiée? D'une part, "On Denoting" introduit un raffinement en établissant une distinction entre les noms propres et les descriptions. D'autre part, et ceci est beaucoup plus important, le parallélisme entre la structure de la phrase et la structure propositionnelle ne tient plus. Lorsqu'une phrase contient une expression dénotante, la théorie du reflet de la structure propositionnelle dans la structure de la phrase ne peut être appliquée qu'après une traduction de la phrase, traduction qui a pour but, justement, d'exhiber les véritables constituants de la proposition. La théorie des descriptions « donne une réduction de toutes les propositions dans lesquelles des expressions dénotantes ont 
une occurrence, à des formes dans lesquelles de telles expressions n'ont pas d'occurrences ${ }^{28}$. Mais une fois que cette réduction a été effectuée, la théorie du parallélisme s'applique à nouveau car « toute pensée doit partir de la fréquentation (. . .) » ${ }^{29}$.

En quoi cette théorie qui, afin d'exhiber les constituants réels des propositions, ces constituants avec lesquels nous avons une "fréquentation immédiate ${ }^{30}$, effectue une traduction des phrases contenant des expressions dénotantes, peut-elle apporter une voie de solution au problème des contradictions?

Il faut tout d'abord remarquer qu'en renonçant au parallélisme strict, la nouvelle théorie rend non signifiantes certaines expressions lorsqu'elles sont prises isolément.

Ce qui apparât ici, c'est l'élucidation du lien entre la dénotation et le statut de la variable. Plus précisément, les mots qui indiquent qu'il y a dénotation comme " tous ", " quelque ", etc., ne sont plus considérés que comme des abréviations linguistiques dans une proposition qui utilise des variables liées. Ces expressions n'ont donc pas de signification : elles sont incomplètes, et cette nouvelle notion d'incomplétude d'une expression va jouer un rôle fondamental par la suite.

\section{LA THÉORIE SUBSTITUTIONNELLE DES CLASSES}

Un mois après la publication de "On Denoting", la London Mathematical Society reçoit un texte de Russell pour publication dans ses Proceedings, intitule "On Some Difficulties in the Theory of Transfinite Numbers and Order Types" 31 .

Dans ce texte, Russell se propose de discuter de deux difficultés que l'on rencontre dans la théorie des ordinaux et des

28. Nos citations de "On Denoting" sont tirées de Essays in Analysis, Lackey, D. (ed.), Londres, 1973, (ci-après E.I.A.). p. 107. L'utilisation par Russell du mot proposition est ici un abus du langage. En effet, une expression dénotante ne peut avoir d'occurrence dans une proposition que si la proposition porte sur une telle expression. Autrement, l'expression dénotante a une occurrence dans la phrase correspondante. Un tel abus n'est justifiable qu'à l'intérieur d'une théorie qui, comme celle des Principles, s'appuie sur un parallélisme strict. Or ici, cette confusion est d'autant plus inexcusable que justement c'est ce parallélisme qui est mis en cause.

29. E.I.A., p. 104 .

30. E.I.A., p. 119.

31. Repris dans E.I.A., p. 135-164. 
cardinaux transfinis. La première de ces difficultés est la contradiction et la seconde concerne l'axiome du choix.

Le texte de Russell est ainsi divisé en trois sections. La première est une analyse des contradictions : l'auteur dégage une matrice universelle pour la génération des paradoxes; la seconde propose trois voies de solution pour éviter les paradoxes en théorie des ensembles ; et la troisième traite de l'axiome du choix. C'est la seconde partie qui retiendra notre attention.

Russell introduit la notion de fonction propositionnelle : soit $\phi ! x$ une expression qui, " pour chaque valeur de $x$, est une proposition ${ }^{32}$. Parallèlement, Russell parle de classe et annonce qu'il va montrer que même si $\phi ! x$ est bien définie pour toute valeur de $x$ (vraie ou fausse) « les valeurs de $x$ pour lesquelles $\phi ! x$ est vraie ne forment pas une classe $»^{33}$ ou, ce qu'il semble voir comme équivalent, on ne peut pas extraire une forme commune $\phi$ à partir d'énoncés $\phi ! x$ car $\phi$ n'est pas une entité. " En d'autres mots, un énoncé à propos de $x$ ne peut pas en général être analysé en deux parties, $x$ et ce qui est dit de $x »^{34}$.

Russell est ainsi amené à distinguer deux sortes de normes ou fonctions propositionnelles : celles qui déterminent des classes et qu'il appelle prédicatives et les autres qu'il appelle non-prédicatives.

Russell envisage trois approches pour éviter la contradiction. Nous ne retiendrons que la troisième : la No-Class Theory ${ }^{35}$.

Brièvement, cette théorie est la suivante. Plutôt que de parler de fonctions $\phi ! x$, ce qui semble suggérer « l'existence de quelque chose dénoté par $\phi{ }^{36}$, on part d'une proposition et

32. E.I.A., p. 136. Nous ne pouvons une fois de plus que souligner l'utilisation abusive que Russell fait des mots expression et proposition. En effet, si $\phi ! x$ est une expression, on voit mal comment l'on peut obtenir une proposition en remplaçant $x$ par quoi que ce soit.

33. E.I.A., p. 137.

34. E.I.A., p. 137.

35. Les deux autres voies sont la " limitation de taille " qui consiste à n'admettre que des fonctions propositionnelles qui définissent des classes pas trop grandes et la "Zig-zag Theory" qui consiste à n'admettre que des fonctions propositionnelles qui sont suffisamment simples. Cette dernière approche est celle qui a servi d'arrière-fond pour la première tentative de solution de la contradiction. Voir $P$., p. 103-104, ainsi que Russell, B., "Les paradoxes de la logique ", in Revue de métaphysique et de morale, 14, (1906), p. 629. Voir également les commentaires de Gödel in Pears, op. cit., p. 200-203.

36. E.I.A., p. 155. 
d'un composant de la proposition. Bien qu'il n'y ait pas d'indications précises à ce sujet, l'entité $a$ doit être quelque chose comme un sujet logique de la proposition, c'est-à-dire un des objets sur lesquels portera la proposition. Russell se contente de parler de constituants de la proposition, c'est-à-dire ce qui est mentionné en énonçant $p$. Ce qui tient alors lieu de fonction propositionnelle est $p(x, a)$ qui est, par définition, ce que $p$ devient lorsque l'on substitue $x$ à $a$ dans $p$. Maintenant, les propositions portant sur une fonction propositionnelle ou sur une classe peuvent être remplacées par d'autres ne contenant ni classe ni fonction propositionnelle. Par exemple, la proposition « La classe $u$ n'a qu'un élément » sera remplacée par «Il y a une entité $b$ telle que $p(x, a)$ est vraie si et seulement si $x$ est identique à $b{ }^{37}$.

Russell voit trois objections à cette théorie, objections qu'il vaut la peine d'examiner de plus près.

La première est « qu'il semble évident pour le sens commun qu'il y a des classes ${ }^{38}$, la seconde que la plus grande partie de l'arithmétique transfinie devient invalide et la troisième est qu'il devient très difficile d'élaborer même l'arithmétique élémentaire.

De ces trois objections, seule la première constitue une objection philosophique : on retrouve là le même problème qu'à l'époque des Principles, à savoir celui de la nature des classes. Et si finalement la classe avait droit de cité dans le "monde de l'être ", une théorie qui s'en passerait, passerait du même coup à côté de quelque chose d'essentiel. Il y a plus. La solution que cherche Russell n'est pas une solution quelconque. En 1959, il écrira :

Tandis que je cherchais une solution, il me sembla qu'il fallait réunir trois conditions pour que la solution fût entièrement satisfaisante. La première de celles-ci, qui était absolument impérative, était la disparition des contradictions. La deuxième (. . .) était que la solution laissât autant que possible les mathématiques intactes. La troisième (. . .) était que, à la réflexion, la solution fît appel à ce que l'on peut nommer le " sens commun

37. E.I.A., p. 155.

38. E.I.A., p. 154. 
logique ", c'est-à-dire qu'en fin de compte, elle semblât exactement ce que l'on devait avoir espéré tout au long ${ }^{39}$.

On remarquera qu'à part la première condition, que toute tentative de solution devra satisfaire, les autres sont, à peu de chose près, les reproches que Russell fait à sa No-Class Theory, c'est-à-dire son caractère artificiel et son incapacité apparente à reconstruire les mathématiques qui utilisent le transfini.

Russell lira son texte devant la Société le 14 décembre mais lorsque celui-ci paraîtra dans les Proceedings, une note aura été ajoutée, datée du 5 février, affirmant que l'auteur n'entretient pratiquement aucun doute que la No-Class Theory soit la bonne solution.

Cette solution, Russell l'élaborera rapidement et le 24 avril 1906, il envoie à la Société un texte intitulé "On the Substitutionnal Theory of Classes and Relations".

Ce texte débute par une présentation succincte des idées développées dans "On Denoting"; Russell expose sa thèse concernant l'absence de signification de certains composants des phrases lorsqu'ils sont isolés du contexte et introduit la notion de «fausses abstractions" (false abstractions) ${ }^{40}$.

La théorie que je veux défendre est que les classes, les relations, les nombres, et en fait presque toutes les choses dont les mathématiques traitent, sont de "fausses abstractions ", dans le sens où « l'actuel roi d'Angleterre » ou « l'actuel roi de France » est une « fausse abstraction " 41 .

Selon Russell, donc, il n'y a pas de classes et tout énoncé portant sur une classe n'est signifiant que s'il peut être analysé en énoncé sur tout ou quelque élément de classe. Formellement, on procède de la façon suivante :

$p(x / a) ! q$ ou $p l a ; x ! q$ signifie \& $q$ résulte de $p$ par substitution de $x$ à $a$ pour toutes ses occurrences dans $p$ ».

39. E.I.A., p. 99 .

40. E.I.A., p. 167 - La notion de fausse abstraction mérite quelques commentaires. D'après les exemples de Russell et ses appels à la théorie des descriptions, une fausse abstraction serait quelque chose comme une pseudo-entité obtenue par une mauvaise application du principe d'abstraction.

41. E.I.A., p. 166. 
Si maintenant nous considérons $p(x / a)$ et $p / a ; x$, nous appelerons $(x / a)$ une substitution et $p l a$ une matrice. On remarquera que substitution et matrice ne sont pas des objets indépendants. "Ainsi la matrice pla est un symbole pour l'expression «le résultat du remplacement de $a$ dans $p$ par », qui est incomplète et sans signification ; pour lui donner une signification, nous devons ajouter le nom d'une entité qui doit remplacer $a \gg{ }^{42}$.

Nous arrivons ici à l'essentiel de la théorie substitutionnelle : ce sont les matrices, symboles pour des expressions incomplètes, qui sont appelées à jouer le rôle de classes. C'est en ce sens que nous pouvons dire qu'il n'y a pas de classes ou, par abus de langage, qu'une classe est un symbole incomplet.

Cette incomplétude des matrices permet d'élaborer une hiérarchie. Plus explicitement, c'est le degré d'incomplétude d'une matrice qui détermine sa place dans la hiérarchie, c'est-à-dire son type.

Le premier type sera ainsi celui des classes d'individus, c'est-à-dire correspondant aux matrices de la forme $(p / a)$.

On voit comment engendrer une hiérarchie croissante de classes et comment intuitivement les contradictions seront évitées. En effet, pour qu'une contradiction du genre de " la contradiction "se manifeste, il faut que quelque part il y ait une boucle, qu'une définition soit circulaire. Or, pour qu'il y ait circularité, il faudrait que quelque part il y ait eu comme argument d'une matrice quelque chose qui ne soit pas du bon type. Nous pouvons toujours construire des expressions vides. "Mais si nous essayons d'interpréter (disons) $\langle p l a=q /(b, c)\rangle$, nous trouvons, en fournissant un $x$, que nous avons une proposition à gauche et une matrice à droite $" 43$.

Comment une théorie qui quelques mois auparavant semblait douteuse, parce que non seulement elle violait le sens commun, mais rendait de plus la reconstruction de l'arithmétique sinon impossible du moins « difficile et compliquée " ${ }^{44}$, com-

42. E.I.A., p. 170.

43. E.I.A., p. 177.

44. E.I.A., p. 156. 
ment donc une telle théorie peut-elle s'imposer non seulement comme la solultion, mais comme une "simplification " ${ }^{45}$ ?

La réponse repose en bonne partie sur le rôle que joue la théorie des descriptions. Dans l'article de 1905, où la No-Class Theory est présentée, aucune allusion n'est en effet faite à la théorie des descriptions. Et ceci n'est pas le fait du hasard. La No-Class Theory n'est pas une théorie sur la manière de construire des expressions ou des phrases sans engendrer de contradiction ; c'est une théorie métaphysique ; c'est une théorie qui tient un discours sur le monde de l'être et non sur le langage ou sur le lien entre le langage et l'être.

L'article de 1906, lui, débute sur une présentation des principes utilisés par la théorie des descriptions, soit sur des techniques de traduction dans une langue logique. Ce "glissement »du métaphysique vers le syntaxique n'est cependant pas dû à une découverte technique.

Ce qui n'apparaît pas naturel en 1905 , c'est que la seule raison qui nous interdit d'affirmer que " ces valeurs forment collectivement une seule entité qui est la classe qu'elles composent $»^{46}$, est que l'application de ce principe conduit quelquefois à des contradictions. La théorie substitutionnelle apporte une justification indépendante à cette interdiction, à tel point que le problème ne se pose même plus. La théorie des descriptions, en transposant sur le plan linguistique (ou si l'on veut, en faisant émerger au niveau linguistique) les problèmes de circularité, naturalise la No-Class Theory, la rend acceptable pour le sens commun logique.

Bref, "les limitations qui rendent les contradictions évitables sont imposées entièrement par le fait que les matrices ne sont pas des entités, avec le besoin d'homogénéité qui s'ensuit $»^{47}$.

Cette homogénéité reste bien sûr celle du monde de l'être ; mais moyennant la théorie des descriptions, cette homogénéité a une traduction syntaxique immédiate. La grande découverte

45. E.I.A., p. 188

46. E.I.A., p. 155

47. E.I.A., p. 188. 
de Russell est de transformer à l'aide de la théorie des descriptions les problèmes de pseudo-entités en pseudo-problèmes d'entités. Il n'y a plus de problèmes à propos des classes car dans une langue bien faite on ne parle pas de classe car il n'y en a pas. Ceci résout pour Russell le vieux problème de l'un et du multiple. La conclusion de l'article de 1906 le confirme :

Sur les conséquences philosophiques de la théorie je ne dirai rien, sinon de relever qu'elle apporte ce qui semble au moins une solution complète de toutes les difficultés vétustes à propos de l'un et du multiple; car même si elle autorise qu'il y ait plusieurs entités, elle fait sienne avec une pédanterie draconnienne la vieille maxime qui dit que : «ce qui est, est un ${ }^{48}$.

Il faut lire qu'il n'y a pas d'objet «brumeux » comme les classes des Principles ; l'introduction de ces pseudo-objets n'était qu'un malentendu dû à une confiance un peu trop grande envers la grammaire superficielle. L'effet premier de la théorie des descriptions est de détruire cette confiance et de proposer une langue logique qui, elle, mérite notre confiance.

La théorie des descriptions que Russell présente comme essentielle à la théorie des types ne joue donc qu'un rôle indirect dans cette dernière. La théorie des types est une théorie sur la structure de l'être, la théorie des descriptions est une théorie sur la traduction dans une langue logique : le lien qui les unit provient du fait que cette langue logique exhibe cette structure.

La No-class Theory et, dans une certaine mesure, la (proto-) théorie des types de 1903, contenaient les idées essentielles à l'élaboration de la théorie des types. Ce qui faisait défaut, c'est le « sens commun » reliant les idées métaphysiques à l'intuition linguistique.

En fournissant ce sens commun, la théorie des descriptions a rendu la typologie acceptable. La théorie des descriptions apparaît donc comme un prétexte philosophique, un support à la théorie métaphysique qu'est la théorie des types.

Russell ne fera pas publier son texte. Quelques mois plus tard, il enverra à la Revue de Métaphysique et de Morale son article intitulé "Les paradoxes de la logique ", où apparaît le "prin-

$\overline{48 . \quad \text { E.I.A., p. } 189 .}$ 
cipe du cercle vicieux $"{ }^{49}$. Dans la formulation même qu'en donne Russell, le déplacement de l'objet d'analyse est perceptible : "Tout ce qui contient une variable doit être exclu des valeurs possibles de cette variable " ${ }^{50}$. La nature du référent de ce tout est définitivement linguistique : "Autrement dit, il faut construire une théorie des expressions contenant des variables apparentes qui fournisse comme conséquence le principe du cercle vicieux " $"$. L'introduction de la No-Class Theory a ainsi pour effet de transformer une théorie sur l'être en une théorie sur l'expression de l'être.

L'introduction de l'axiome de réductibilité, qui permet d'affirmer l'existence d'une fonction prédicative coextensive à une fonction d'un ordre quelconque, est à cet égard exemplaire. En effet, pour éviter la construction d'entités contradictoires, la théorie des types conservait aux objets la trace de leur construction. Mais cette trace est-elle linguistique ou ontologique? L'axiome de réductibilité semble trancher en faveur du caractère syntaxique, car ayant pour effet d'effacer partiellement cette trace, n'affirme-t-il pas l'inanité, ontologiquement parlant, de la hiérarchie elle-même ${ }^{52}$ ?

À son départ, l'entreprise russellienne de reconstruction logique des mathématiques apparaît donc comme une entreprise quasi empirique. Bien sûr, les entités auxquelles se confronte le philosophe ne sont pas des atomes ou des données des sens. Ce sont les entités, celles qui ont droit de cité et dont la combinatoire rend compte de ce qui est. N'a-t-il pas qualifié lui-même son entreprise, dans la préface des Principles, de « processus ana-

49. "Les paradoxes de la logique ", op. cit., p. 627-650. Cet article est une réponse à H. Poincaré, "Les Mathématiques et la Logique ", Revue de métaphysique et de morale 14, (1906), p. 294-317. Poincaré emprunte l'expression " cercle vicieux " à Richard.

50. "Les paradoxes de la logique ", op. cit., p. 641.

51. Idem, p. 642.

52. Il y aurait beaucoup à dire sur l'axiome de réductibilité, en opposant son contenu philosophique à son contenu opératoire ; mais cela dépasse le cadre de cet article où nous voulons nous restreindre aux conditions d'émergence de la théorie des types. Remarquons cependant que si, comme nous croyons l'avoir montré, l'introduction de la No-Class Theory se justifie par son aspect naturel ", c'est-à-dire qu'elle fait " appel à ce qu'on peut nommer le "sens commun logique " ", l'introduction de l'axiome de réductibilité repose, elle, sur le besoin de laisser " autant que possible les mathématiques intactes". Le lecteur intéressé à ces questions consultera : Quine, W. V. "Russell's Theory of Types", in Klemke, op. cit., p. 383, et surtout Gödel, K., "Russell's Mathematical Logic", in Pears, op. cit., p. 213. 
logue à celui qui a abouti à la découverte de Neptune, à la différence que l'étape finale - la recherche avec un télescope mental des entités qui ont été inférées - est souvent la partie la plus difficile de l'entreprise $" 53$.

Le télescope ici sera la théorie des descriptions qui, par sa technique de traduction, permettra de révéler les véritables entités qui composent les propositions.

Le système philosophique élaboré dans les Principles reste, du moins dans son aspect philosophie du langage, assez naif. Bien sûr, pratiquement toute philosophie du langage a pour but de rendre compte de ce pouvoir qu'a le langage de représenter le monde. À l'instar de beaucoup d'autres, Russell choisit la grammaire comme guide, ce qui le conduit à une analyse relativement simple, voire triviale. Mais cette trivialité n'est pas contraignante ; le problème des contradictions, qui est un problème universel, c'est-à-dire non lié à un système philosophique particulier, saura y trouver une solution. Et cette solution, la théorie des types, sera suffisamment forte pour apparaître encore aujourd'hui à certains comme la solution ${ }^{54}$.

Département de philosophie

Université du Québec à Montréal

53. P., p. XV.

54. Je fais ici allusion aux travaux contemporains qui font un usage non-trivial de la théorie des types, par exemple en sémantique des langues naturelles dans ce qu'il est maintenant convenu d'appeler " les grammaires de Montague ». 